\title{
Weather dependent estimation of continent-wide wind power generation based on spatio-temporal clustering
}

\author{
Bruno U. Schyska ${ }^{1,2}$, António Couto ${ }^{3}$, Lueder von Bremen ${ }^{1,2}$, Ana Estanqueiro ${ }^{3}$, and \\ Detlev Heinemann ${ }^{1,2}$ \\ ${ }^{1}$ Institute of Physics, Energy Meteorology group, University of Oldenburg, Oldenburg, Germany \\ ${ }^{2}$ ForWind Center for Wind Energy Research, University of Oldenburg, Oldenburg, Germany \\ ${ }^{3}$ Laboratório Nacional de Energia e Geologia, Energy Analysis and Networks Unit, Lisboa, Portugal \\ Correspondence to: Bruno U. Schyska (bruno.schyska@uni-oldenburg.de)
}

Received: 19 December 2016 - Revised: 30 March 2017 - Accepted: 10 April 2017 - Published: 29 May 2017

\begin{abstract}
Europe is facing the challenge of increasing shares of energy from variable renewable sources. Furthermore, it is heading towards a fully integrated electricity market, i.e. a Europe-wide electricity system. The stable operation of this large-scale renewable power system requires detailed information on the amount of electricity being transmitted now and in the future. To estimate the actual amount of electricity, upscaling algorithms are applied. Those algorithms - until now - however, only exist for smaller regions (e.g. transmission zones and single wind farms). The aim of this study is to introduce a new approach to estimate Europe-wide wind power generation based on spatio-temporal clustering. We furthermore show that training the upscaling model for different prevailing weather situations allows to further reduce the number of reference sites without losing accuracy.
\end{abstract}

\section{Introduction}

A fully integrated European energy market is one of the priority policy areas of the European Commission (e.g. EC, 2016). Transmission system operators use estimates of the energy production from variable renewable sources within their transmission zones already today. Besides technical aspects, such as the reinforcement of the transmission grid (e.g. Becker et al., 2014; Kies et al., 2016b), also the upscaling algorithms behind these renewable power estimates need to be revised when trading zones are extended - in particular for increasing shares of renewables. In fact, the large-scale integration of variable renewable energy sources (VRES) such as wind power - introduces additional factors of uncertainty. This uncertainty poses new challenges to the power system operator since it is necessary to keep the balance between production and consumption at every moment, in order to ensure the stability of the power system (Holttinen et al., 2011; Pérez-Arragia and Batlle, 2012; Estanqueiro, 2008). In this sense, it is crucial to know the actual and future generation from the VRES within the system. While the future gen- eration is subject of forecasting technologies, this work focuses on the introduction of an upscaling methodology to estimate the Europe-wide actual wind power generation based on spatio-temporal clustering (e.g. Kisilevich et al., 2010).

With the application of upscaling methodologies on the European scale additional potential benefits are expected: Aggregating wind parks with a wide geographical dispersion, for instance, is an effective way to reduce the short term variability and forecast errors by taking advantage of the statistical smoothing effect (Liu et al., 2014; Miettinen et al., 2014; Marrone et al., 2008).

In the current literature, several upscaling approaches can be found: In Ishihara et al. (2007) a typical upscaling function using a bi-exponential function to estimate the crosscorrelation is proposed. Pinson et al. (2003) performed a benchmarking of different approaches based on dynamic fuzzy neural networks. In Lobo and Sanchez (2012) the upscaling technique is based on smoothing techniques to construct the predictions of the aggregated wind generation from historical wind speed predictions and the associated wind generation measurements. Recently, Li et al. (2015) proposed 
a probabilistic approach showing that this type of methodology can provide competitive interval forecasts when compared to conventional statistical approaches. However, all of the upscaling methodologies described above are usually applied to a set of wind parks, and not to the European scale.

As wind is a meteorological quantity, weather conditions may have a strong impact on the wind power variability as well as on the uncertainty of its forecasts (Giebel et al., 2011; Ernst et al., 2007). Lange and Heinemann (2003) for instance show that the presence of cyclonic systems with strong dynamics - such as cold fronts - can be related to larger errors in the forecast when compared with prevailing weather conditions associated with stationary systems such as anticyclonic systems. A similar methodology was also applied to several wind parks in Portugal demonstrating the weather dependency of the wind power forecast errors (Trancoso, 2012). Vincent (2010) shows that strong wind variability can be associated with certain weather patterns and Couto et al. (2015) show a strong impact of weather regimes on wind power ramps in Portugal. Consequently, taking into account the underlying role of the synoptic weather patterns could be an important step towards reliable upscaling algorithms.

The objective of this work is to introduce a new upscaling approach for Europe-wide wind power generation based on spatio-temporal clustering (Sect. 2.1). The upscaling model will be trained and evaluated for different circulation weather types (CWTs, Sect. 2.2) using a set of Europe-wide wind power generation data (Sect. 2.3). The training for specific CWTs will be compared to the training over all time steps in the training period in order to investigate its weather dependency and the potential benefit from the weather dependent training (Sect. 3). Conclusions will be drawn in Sect. 4.

\section{Methodology and data}

\subsection{Reference site selection: spatio-temporal clustering}

Focus of this work is the presentation of a reference site selection scheme based on spatio-temporal clustering. In order to derive a finite set of reference sites to upscale the generation of wind power across Europe at a certain point of time the following procedure is applied:

1. Cluster the locations of wind farms (latitude/longitude coordinates) into $N$ (geographical) clusters via the kmeans algorithm (Mac Queen, 1967).

2. For each of the $N$ geographical clusters, select the site with the highest wind power capacity. Obtain the set $\Omega_{\text {geo }}$ with size $\left|\Omega_{\text {geo }}\right|=N$.

3. Compute pairwise (temporal) correlations $\varrho\left(r_{i}, r_{j}\right)=$ $\varrho\left(p\left(r_{i}, t\right), p\left(r_{j}, t\right)\right) \forall r_{i}, r_{j} \in \Omega_{\mathrm{geo}}$ of the historical generation time series $p\left(r_{i}, t\right)$ at the $N$ sites $r_{i}, i=1, \ldots N$ selected in the previous step.

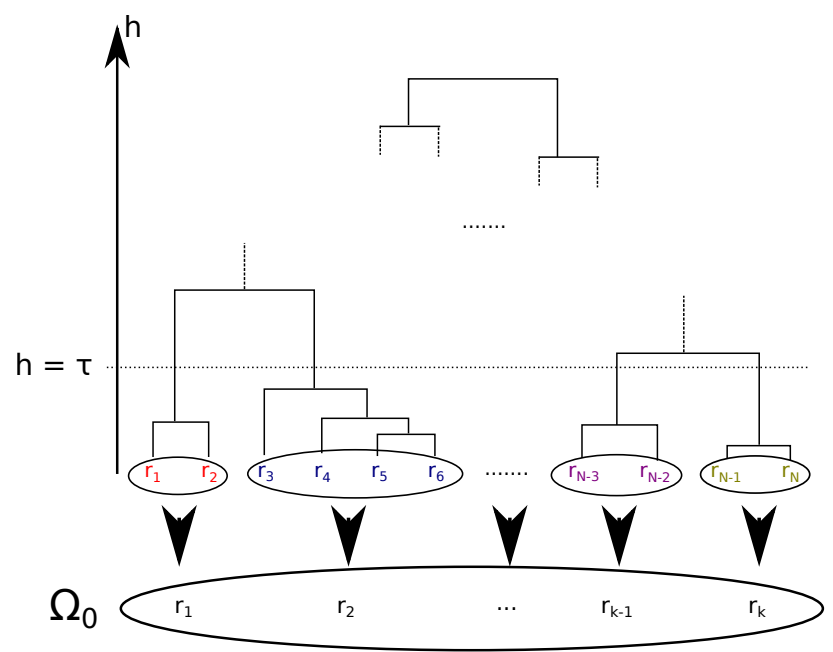

Figure 1. Schematic dendrogram for illustration of steps 4 and 5 of the spatio-temporal clustering approach.

4. Use the correlation information to apply a hierarchical clustering (e.g. Rockach, 2010) with the distance between sites $r_{i}$ and $r_{j}$ being defined as $d\left(r_{i}, r_{j}\right)=$ $1-\left|\varrho_{i j}\right|$.

5. Cut the dendrogram obtained from the hierarchical (temporal) clustering at height $h=\tau$. Yield $k=k(\tau) \leq$ $N$ clusters. Here, $\tau$ is the distance between two clusters. For each cluster, again, select the site with the highest wind power capacity as cluster centres to obtain the final set of $k$ reference sites $\Omega_{0}$. This step is illustrated in Fig. 1.

Note that if the average group linkage method is used to agglomerate clusters, $\tau$ can be interpreted as 1 minus the average intra-cluster correlation. In other words, the final set of reference sites can be determined by choosing the average intra-cluster correlation:

$$
\begin{aligned}
D(A, B) & :=\frac{1}{(|A|+|B|)(|A|+|B|-1)} \sum_{x, y \in A \cup B} d(x, y) \\
& =\frac{1}{(|A|+|B|)(|A|+|B|-1)} \sum_{x, y \in A \cup B}(1-\varrho(x, y)) \\
& =1-\bar{\varrho}_{C}
\end{aligned}
$$

For two clusters (sets) $A$ and $B$ and $C=A \cup B$, i.e. the cluster resulting from the union of set $A$ and set $B$. Choosing the average intra-cluster correlation as key-parameter to determine the reference sites allows to further investigate the behavior of the clustering approach from a physical-meteorological perspective. This is the major advantage of the proposed methodology compared to, for instance, st-DBSCAN (Birant and Kut, 2007), which does not allow for using different distance measures than the euclidean distance. 


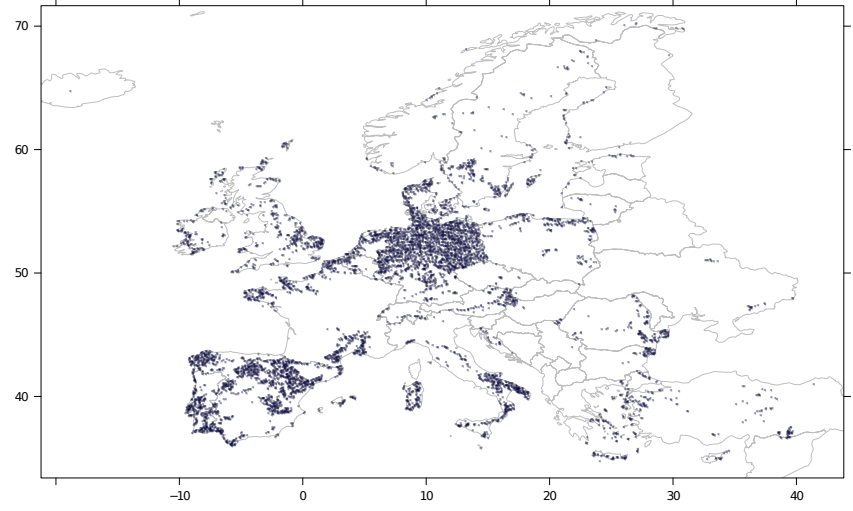

Figure 2. Modelled spatial distribution of rated wind power capacity across Europe.

\subsection{Upscaling and evaluation}

The upscaling estimate itself for time $t=t^{\prime}$ is computed as a weighted sum of the generation measured at the reference sites:

$E\left(t=t^{\prime}\right)=\sum_{r_{i} \in \Omega_{0}} w\left(r_{i}\right) p\left(r_{i}, t=t^{\prime}\right)$

Where the weights $w_{i}$ are computed from a multiple linear regression of the generation at the $k$ reference sites $r_{k} \in \Omega_{0}$ on the total Europe-wide generation performed over a prechosen training period. Note, that in general the $w_{i}$ may vary in dependency of $\tau$ and $N$.

For this study, the upscaling estimate derived from Eq. (2) will be evaluated based on the Pearson correlation and the root mean square error (RMSE) between the upscaling estimate $E(t)$ and the reference time series for a testing period. Here, the sum of all grid cells of the wind power generation data (Sect. 2.3) is used as reference. RMSE values have been normalized to the average hourly wind power production.

In order to investigate the dependency from the prevailing weather situation and the eventual benefit from training the model for specific weather situations, both training and testing will be performed for the nine most common circulation weather types in Europe (see Sect. 2.4).

We use five years (2008-2012) for training and one year (2013) for testing.

\subsection{Wind power generation data}

The upscaling methodology introduced above is tested for a data set of modeled hourly onshore wind power generation across Europe. This data bases on two data sets: COSMOEU analysis data provided by the German Weather Service (Doms et al., 2011) used for the statistical downscaling of MERRA reanalysis data provided by the National Aeronautics and Space Administration of the United States (Bosilovich, 2008). MERRA was used to capture a longer period of time.
The spatial distribution of rated wind power across Europe is modeled as a function of the average (computed over the period considered) wind speed for each location (grid cell) in Europe. The relation between wind speed and rated power is estimated based on the available data of deployed wind power capacity in Germany. Since this relation is not very distinct, artificial noise has additionally been added:

$y(r)=a \bar{w}(r)+b+\varepsilon$

Here, $y(r)$ is the rated wind power at location $r, \bar{w}(r)$ is the average wind speed at the same location, $a$ and $b$ are coefficient and intercept fitted from the available data and $\varepsilon$ is artificial gaussian noise with zero mean.

The spatial distribution is shown in Fig. 2. Note, that it does not - and is not meant to - represent the real spatial distribution. Furthermore, offshore locations are not included.

Wind speed is converted to wind power by applying the regional power curve model for the largest German transmission zone developed by Späth et al. (2015). The procedure described here is similar to the one used by Kies et al. (2016a). For this study, the years 2008-2013 are considered.

\subsection{Circulation weather types}

Classification of atmospheric circulation into distinct states is a widely used tool for describing and examining weather patterns and their impact on meteorological phenomena, e.g., rainfall (Philipp et al., 2010). In the literature, several methodologies of weather circulation classification are available (Jenkinson and Collinson, 1977; Huth et al., 2008; Philipp et al., 2010; Couto et al., 2015). In this study, an automatic version of the Lamb weather type classification is applied to MERRA sea level pressure fields in order to obtain a time series of prevailing circulation weather types. This method was initially proposed by Jenkinson and Collinson (1977) and thereafter applied by several authors (e.g., Trigo and da Camara, 2000; Costa et al., 2006).

The algorithm bases on the sea level pressure at the 16 points depicted in Fig. 4. Assuming geostrophic conditions, westerly and southerly winds can be computed from the meridional and zonal pressure gradient respectively. Doing so, six circulation indices (southerly flow SF, westerly flow WF, resultant flow FT, southerly shear vorticity ZS, westerly shear vorticity ZW and total shear vorticity ZT) can be computed from the sea level pressure data via:

$$
\begin{aligned}
\mathrm{SF} & =A \cdot \frac{1}{4} \cdot\left(p_{5}+2 p_{9}+p_{13}-p_{4}-2 p_{8}-p_{12}\right) \\
\mathrm{WF} & =\frac{1}{2} \cdot\left(p_{12}+p_{13}-p_{4}-p_{5}\right) \\
\mathrm{FT} & =\sqrt{\mathrm{SF}^{2}+\mathrm{WF}^{2}} \\
\mathrm{ZS} & =B \cdot \frac{1}{4} \cdot\left(p_{6}+2 p_{10}+p_{14}-p_{5}-2 p_{9}-p_{13}\right. \\
& \left.-p_{4}-2 p_{8}-p_{12}+p_{3}+2 p_{7}+p_{11}\right)
\end{aligned}
$$



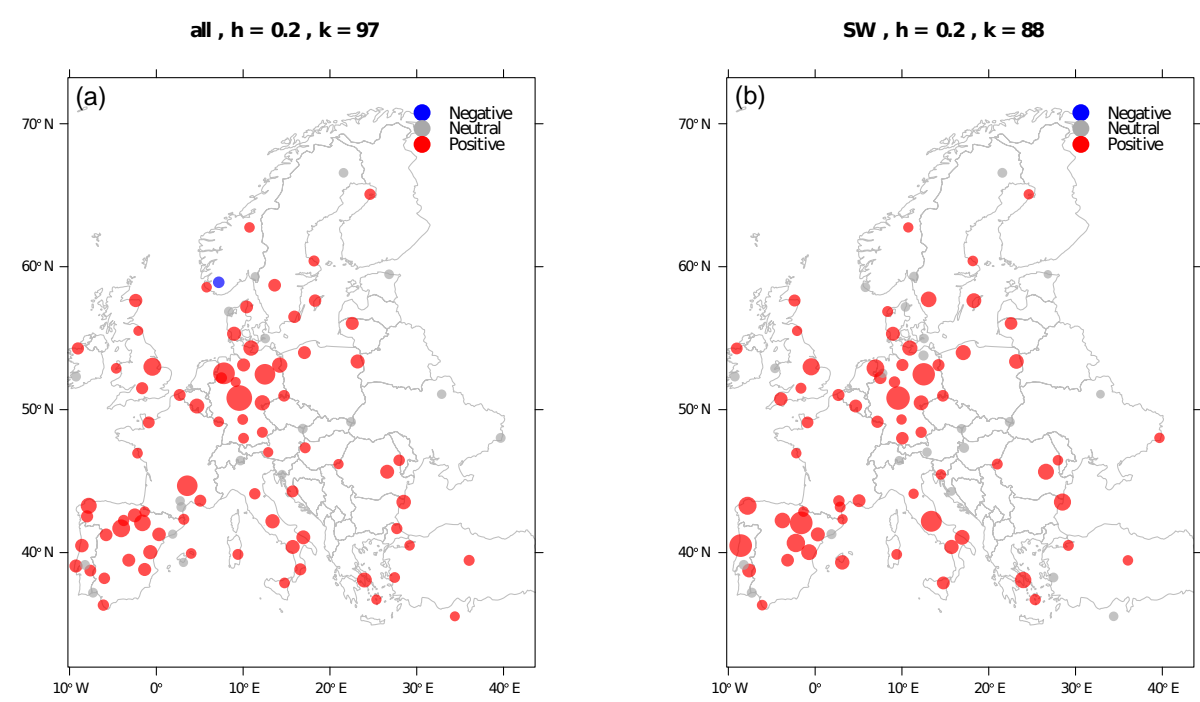

Figure 3. Location of the cluster centres and the weights assigned to them by the linear regression (size scale) for $\bar{\varrho}_{C}=0.8(\hat{=} \tau=0.2)$ and training over all time steps (a) and over the time steps with prevailing CWT SW (b).

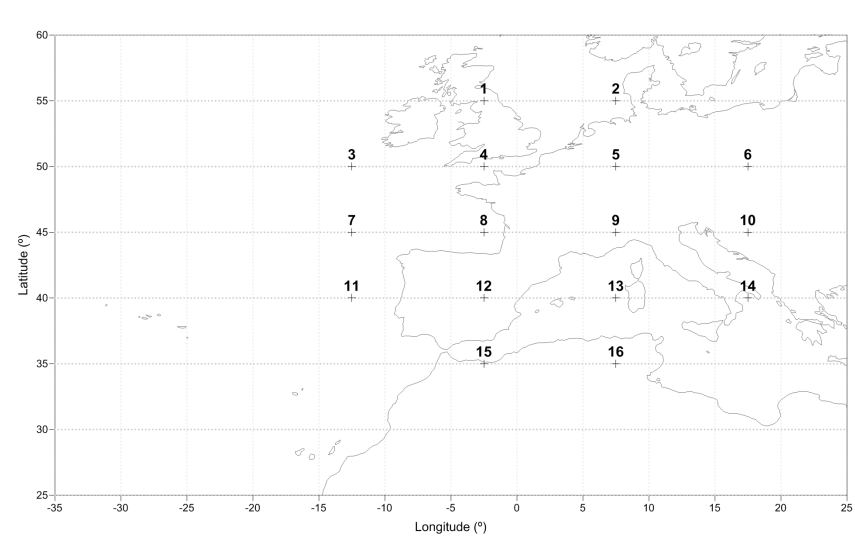

Figure 4. Locations of the 16 points used for the circulation weather type identification.

$$
\begin{aligned}
\mathrm{ZW} & =C \cdot \frac{1}{4} \cdot\left(p_{15}+p_{16}-p_{8}-p_{9}\right)- \\
D & \cdot \frac{1}{4} \cdot\left(p_{8}+p_{9}-p_{1}-p_{2}\right) \\
\mathrm{ZT} & =\mathrm{ZS}+\mathrm{ZW}
\end{aligned}
$$

Southerly and westerly shear vorticity are estimated from the wind shear in the center of the domain. Subscribed numbers indicate the location. The four coefficients $A, B, C$ and $D$ are determined by the central latitude of the chosen raster $\varphi_{0}$ (here: $\varphi_{0}=45^{\circ}$ ):

$$
\begin{aligned}
A & =\frac{1}{\cos \left(\varphi_{0}\right)} \\
B & =\frac{1}{2 \cos ^{2}\left(\varphi_{0}\right)} \\
C & =\frac{\sin \left(\varphi_{0}\right)}{\sin \left(\varphi_{0}-5^{\circ}\right)}
\end{aligned}
$$

$$
D=\frac{\sin \left(\varphi_{0}\right)}{\sin \left(\varphi_{0}+5^{\circ}\right)}
$$

From the six circulation indices 26 circulation weather types (CWTs) can be deduced as follows:

- If $|\mathrm{ZT}|<$ FT the mean flow dominates over the vorticity (local curvature of the wind field). These CWTs are called directional and named after the eight directions North (N), Northeast (NE), East (E), Southeast (SE), South (S), Southwest (SW), West (W) and Northwest $(\mathrm{NW})$. The flow direction is given by $\tan ^{-1} \frac{\mathrm{WF}}{\mathrm{SF}}$ if $\mathrm{WF} \leq 0$ and $\tan ^{-1} \frac{\mathrm{WF}}{\mathrm{SF}}+180^{\circ}$ if $\mathrm{WF}>0$, respectively.

- If $|\mathrm{ZT}|>2 \mathrm{FT}$ the vorticity exceeds the mean flow. The circulation is either cyclonic (L) if ZT $>0$ or anticyclonic $(\mathrm{H})$ if $\mathrm{ZT}<0$

- If FT $<|\mathrm{ZT}|<2 \mathrm{FT}$ both, vorticity and mean flow, are equally strong. These CWTs are called hybrid and named after the prevailing circulation, i.e. either cyclonic or anticyclonic, plus one of the eight flow directions.

For this study, the nine most common CWTs in Europe are chosen for evaluation. These are the directional types except for Southeast, the cyclonic type and the anticyclonic type.

\section{Results}

\subsection{Cluster centres and reference site weights}

As mentioned above, the number of reference sites varies in dependency of the chosen average intra-cluster correlation. Figure 3 shows the locations of the reference sites obtained from the spatio-temporal clustering exemplary for the training over all time steps (a) and the time steps with prevailing 


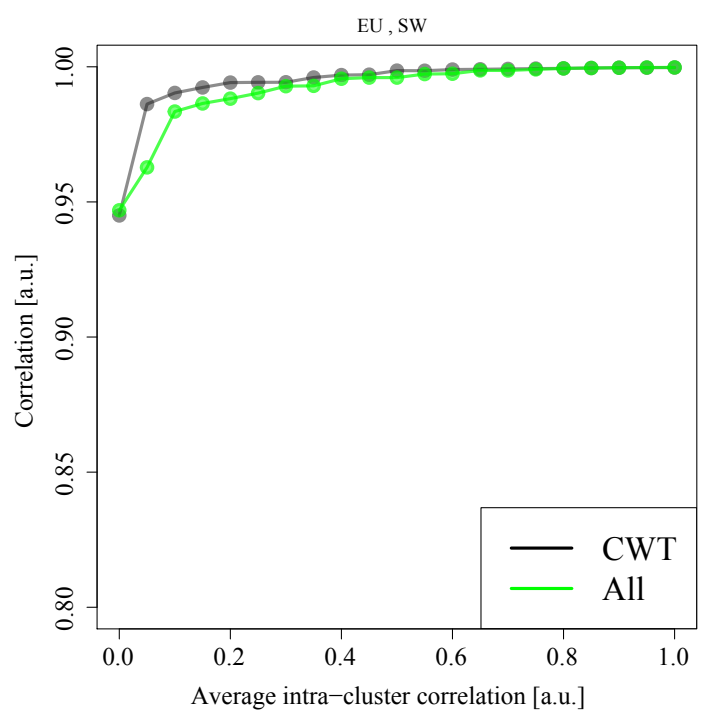

Figure 5. Correlation versus the average intra-cluster correlation $\bar{\varrho}_{\mathrm{C}}$ for CWT SW obtained from the specific training for this CWT (black) and from the training over all time steps (green) respectively.

Southwestern circulation type (b). The average intra-cluster correlation was exemplary set to $\bar{\varrho}_{\mathrm{C}}=0.8$. The size of the dots additionally indicates the weights given to the reference sites by the linear regression. Points with $|w(r)|<0.5 \times \sigma$ are considered as neutral. Here, $\sigma$ denotes the standard deviation computed from all weights.

Obviously, the number of reference sites for the CWT SW (3 right) is lower (88 to 97). Hence, the correlations of wind power generation at the geographical clusters is higher than average during time steps of Southwesterly flow - especially on the Iberian Peninsula where the reduction of reference sites is most apparent. Here, wind power production exhibits a relatively coherent spatial structure. This can be related to the passage of large-scale atmospheric phenomena associated with southwesterly circulation, such as cold fronts, able to cover the whole region (Jim et al., 2009; Peña et al., 2011). However, not all of the nine CWTS considered exhibit this higher-than-average correlation. In contrary to southwesterly circulation, some CWTS are usually associated with relatively weak and diffused synoptic scale phenomena. These may cause a less coherent spatial structure of the wind field. Therefore, the number of reference sites for $\bar{\varrho}_{\mathrm{C}}=0.8$ ranges between 88 for SW and 105 for the Easterly flow type (not shown).

From Fig. 3 it can also be seen, that the weights given to the selected reference sites vary as well. The reference sites on the Iberian Peninsula get relatively higher weights for the Southwesterly circulation type than for all time steps.

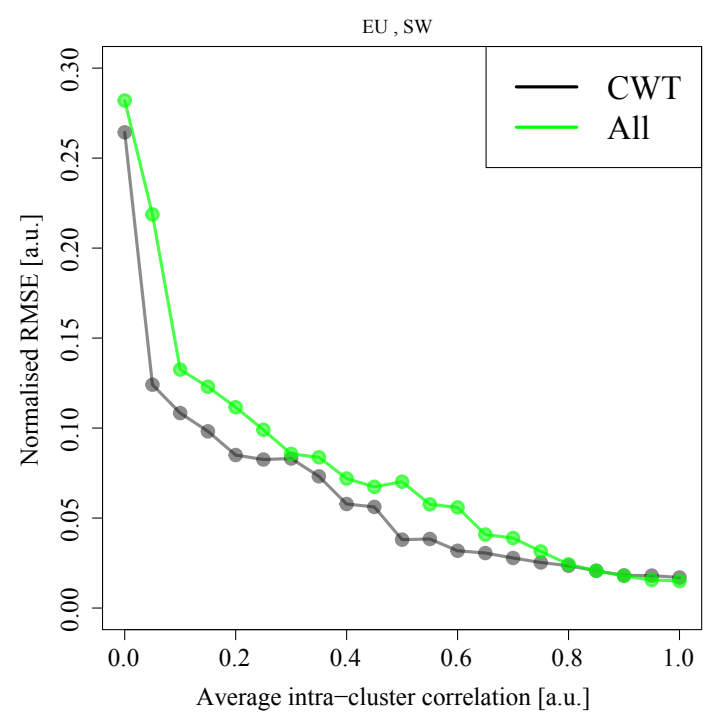

Figure 6. As Fig. 5 but for the RMSE normalised to the average generation.

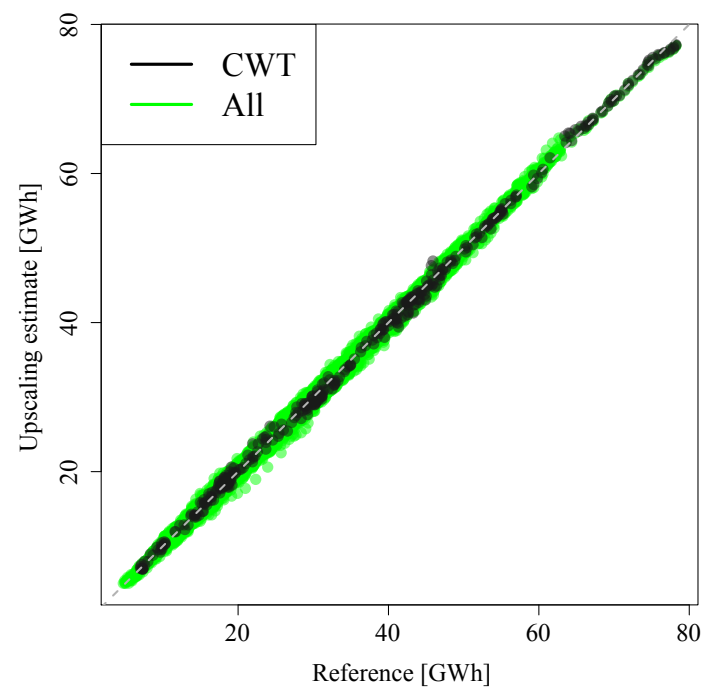

Figure 7. Time series of the upscaling estimate [GWh] versus the reference time series [GWh] for all time steps (green) and time steps with prevailing Southwesterly circulation (black).

\subsection{Upscaling evaluation}

The skill of the methodology introduced in Sect. 2 measured by correlation and RMSE is exemplary shown in Figs. 5 and 6 for the Southwesterly circulation type. It can be seen, that very high $(>0.95)$ values for the correlation can be achieved for average intra-cluster correlations above 0.1 . For the Southwesterly CWT this corresponds to a number of reference sites $k=17$ for whole Europe. For higher $\bar{\varrho}_{C}$ the correlation asymptotically approaches 1 .

A similar behaviour is found for the RMSE. For $\bar{\varrho}_{C}>0.1$ the RMSE drops below $10 \%$ of the average wind power 


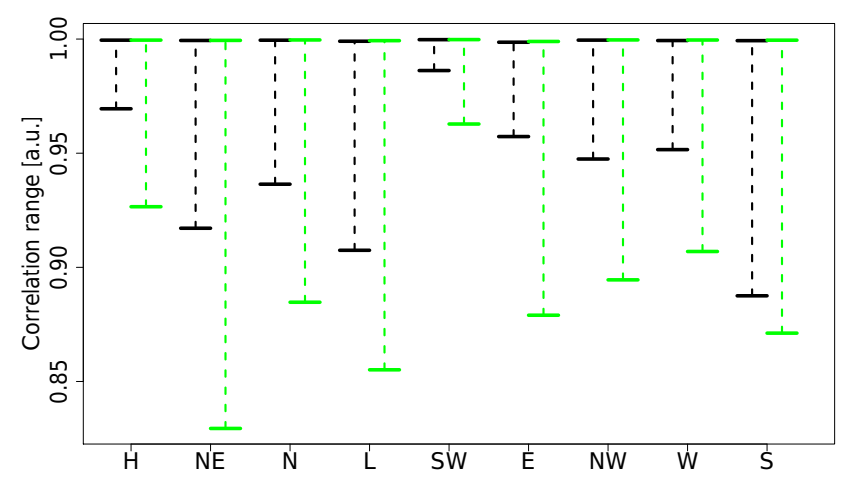

Figure 8. Range of correlation values achieved by training the upscaling for the specific CWTs (black) and from training over all time steps (green).

generation in Europe. For average intra-cluster correlations above 0.45 - corresponding to $k=41-$ RMSE values below $5 \%$ of the average generation can be achieved.

The good agreement between the upscaling estimate and the reference time series can additionally be seen from the scatter plot (Fig. 7, again for $\bar{\varrho}_{\mathrm{C}}=0.8$ ). A systematic error only appears for extreme high (above $75 \mathrm{GWh}$ ) wind power generation values. Here, the upscaling model systematically underestimates the generation. Furthermore, all these extreme values occur during Southwesterly circulations. This reduces the skill of the upscaling model for this CWT disproportionately strong.

$\bar{\varrho}_{\mathrm{C}}=0$ does not involve any hierarchical clustering. The corresponding data point is considered as non-representative and therefore neglected from the further analysis.

\subsection{Benefit from training for weather types}

In general, the Southwesterly CWT is the one, for which the introduced upscaling methodology works best with respect to the correlation (Fig. 8, black bars). Other CWTs exhibit lower correlations. With respect to the RMSE, the SW type only skills average (Fig. 9, black bars). Here, especially the Easterly type benefits from the specific training.

Figures 8 and 9 show the range of the correlation and the RMSE for all $\left.\left.\bar{\varrho}_{C} \in\right] 0,1\right]$ obtained from (i) the training specifically for the particular CWTs in black and (ii) training over all time steps in green. Evidently, the upscaling skill benefits from the specific training. The range of both, correlation and RMSE, can be reduced significantly. It can furthermore be observed that the cyclonic CWT and the Southerly CWT perform worst - with respect to both correlation and RMSE - while the Easterly, Southwesterly and Cyclonic type perform best. The benefit from the CWT specific training is strongest for the Northeasterly and Northwesterly type with respect to correlation and RMSE, respectively.

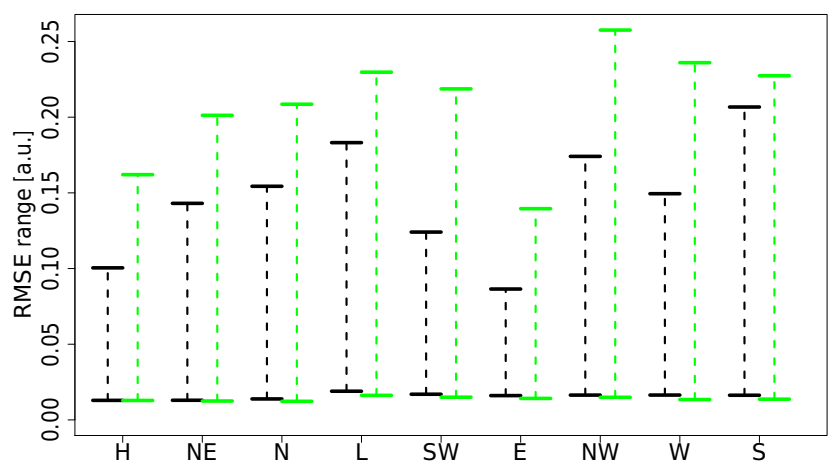

Figure 9. As Fig. 8 but for the RMSE normalised to the average generation.

\section{Discussion and conclusions}

In order to derive a reduced set of reference sites to estimate Europe-wide wind power production, a new spatio-temporal clustering approach has been developed. To test the methodology, model data is used, which is known to be smoother than measured data. Keeping this in mind, we have shown that a rather low number of around 40 reference sites - when chosen carefully - is sufficient to estimate the actual wind power generation across whole Europe with adequate accuracy. We have also shown that it is beneficial to train the upscaling model for different prevailing circulation weather types.

Data availability. All underlying research data of this work is publicly available.

Competing interests. The authors declare that they have no conflict of interest.

Acknowledgements. This work is part of task 82.2 "Upscaling: Minimum reference and data selection and decomposition" of the Integrated Research Programm Wind Energy (IRPWind) conjointly executed by ForWind Oldenburg, LNEG and Fraunhofer IWES. IRPWind has received funding from the European Union's Seventh Programme for research, technological development and demonstration under grant agreement no. 609795. We thank M. Greiner and one anonymous referee for helpful comments to this manuscript. We acknowledge the Ministry of Science and Cultur of Lower Saxony (Germany) for supporting the research project ventus efficiens (ZN3024, MWK Hannover).

Edited by: S.-E. Gryning

Reviewed by: M. Greiner and one anonymous referee 


\section{References}

Becker, S., Rodriguez, R. A., Andresen, G. B., Schramm, S., and Greiner, M.: Transmission grid extensions during the build-up of a fully renewable pan-European electricity supply, Energy, 64, 404-418, 2014.

Birant, D. and Kut, A.: ST-DBSCAN - an algorithm for clustering spatial-temporal data, Data and Knowledge Engineering, 60, 208-2021, 2007.

Bosilovich, M.: Nasa's modern-era retrospective analysis for research and applications: Integrating earth observations, Tech. rep., Earthzine, 2008.

Costa, P., Estanqueiro, A., and Miranda, P.: Building a wind atlas for mainland Portugal using a weather type classification, European Wind Energy Conference and Exhibition, 2006.

Couto, A., Costa, P., Rodrigues, L., Lopes, V. V., and Estanqueiro, A.: Impact of Weather Regimes on the Wind Power Ramp Forecast in Portugal, IEEE Trans. Sustain. Energy, 6, 934-942, 2015.

Doms, G., Schättler, U., and Baldauf, M.: A description of the nonhydrostatic regional cosmo model, part i: Dynamics and numerics, Tech. rep., Consortium for Small-Scale Modelling, 2011.

EC: http://ec.europa.eu/priorities/energy-union-and-climate/ fully-integrated-internal-energy-market_en, last access: 22 September 2016.

Ernst, B., Oakleaf, B., Ahlstrom, M. L., Lange, M., Moehrlen, C., Lange, B., Focken, U., and Rohrig, K.: Predicting the wind, IEEE Power Energ. M., 5, 78-89, 2007.

Estanqueiro, A.: Impact of Wind Generation Fluctuations in the Design and Operation of Power Systems, Proceedings of the 7th international Workshop on Large Scale Integration of Wind Power and on Transmission Networks for Offshore Wind Farms, 2008.

Giebel, G., Brownsword, R., Kariniotakis, G., Denhard, M., and Draxl, C.: The State-Of-The-Art in Short-Term Prediction of Wind Power - A Literature Overview, Tech. Report, ANEMOS.plus, 1-109, 2011.

Holttinen, H., Meibom, P., Orths, A., Lange, B., O’Malley, M., Tande, J. O., Estanqueiro, A., Gomez, E., Söder, L., Strbac, G., Smith, J. C., and van Hulle, F.: Impacts of large amounts of wind power on design and operation of power systems, results of IEA collaboration, Wind Energy, 14, 179-192, 2011.

Huth, R., Beck, C., Philipp, A., Demuzere, M., Ustrnul, Z., Cahynová, M., Kyselý, J., and Tveito, O. E.: Classifications of atmospheric circulation patterns: recent advances and applications, Ann. NY Acad. Sci., 1146, 105-152, 2008.

Ishihara, T., Yamaguchi, A., Ogawa, T., Sakai, K., and Fujino, T.: An upscaling approach for the regional wind power forecasting, European Wind Energy Conference (EWEC), 2007.

Jenkinson, A. F. and Collinson, B. P.: An initial climatology of gales over the North Sea, Synop. Climatol., Branch Memo (62), 1977.

Jim, P. A., Gonz, J. F., Mont, J. P., and Garc, E.: Climatology of wind patterns in the northeast of the Iberian Peninsula, Int. J. Climatol., 29, 501-525, 2009.

Kies, A., Schyska, B. U., and von Bremen, L.: Curtailment in a Highly Renewable Power System and Its Effect on Capacity Factors, Energies, 9, https://doi.org/10.3390/en9070510, 2016a.

Kies, A., Schyska, B. U., and von Bremen, L.: The optimal share of wave power in a highly renewable power system on the Iberian Peninsula, Energy Reports, 2, 221-228, 2016b.
Kisilevich, S., Mansmann, F., Nanni, M., and Rinzivillo, S.: Spatiotemporal clustering, in: Data mining and knowledge discovery handbook, edited by: Maimon, O. and Rockach, L., 2 Edn., 855874, Springer, New York, 2010.

Lange, M. and Heinemann, D.: Relating the uncertainty of shortterm wind speed predictions to meteorological situations with methods from synoptic climatology, Proceedings of the European Wind Energy Conference (EWEC), 2003.

Li, P., Guan, X., and Wu, J.: Aggregated wind power generation probabilistic forecasting based on particle filter, Energy Convers. Manage., 96, 579-587, 2015.

Liu, Y., Gao, X., Yan, J., Han, S., and Infield, D. G.: Clustering methods of wind turbines and its application in short-term wind power forecasts, J. Renew. Sustain. Energy, 6, 053119, https://doi.org/10.1063/1.4898361, 2014.

Lobo, M. G. and Sanchez, I.: Regional wind power forecasting based on smoothing techniques, with application to the Spanish peninsular system, IEEE T. Power Syst., 27, 1990-1997, 2012.

Mac Queen, J. B.: Some methods for classification and analysis of multivariat observations, Proceedings of the 5th Berkeley Symposium on Mathematical Statistics and Probability, University of California Press, Los Angeles, 281-297, 1967.

Marrone, C. A. Q., Knorr, K., Lange, B., and Beyer, H. G.: Characterization and modeling of the variability of the power output of aggregated wind farms, Proceedings of the 7th International Workshop on Large Scale Integration of Wind Power and on Transmission Networks for Offshore Wind Farms Characterization, 2008.

Miettinen, J., Holttinen, H., and Giebel, G.: Nordic Wind Power Forecast Errors: Benefits of Aggregation and Impact to Balancing Market Volumes, Proceedings of the 13th International Workshop on Large-Scale Integration of Wind Power into Power Systems as well as on Transmission Networks for Offshore Wind Plants, 2014.

Peña, J. C., Aran, M., Cunillera, J., and Amaro, J.: Atmospheric circulation patterns associated with strong wind events in Catalonia, Nat. Hazards Earth Syst. Sci., 11, 145-155, https://doi.org/10.5194/nhess-11-145-2011, 2011.

Pérez-Arriaga, I. and Batlle, C.: Impacts of intermittent renewables on electricity generation system operation, Econ. Energy Environ. Policy, 1, 2, https://doi.org/10.5547/2160-5890.1.2.1, 2012.

Philipp, A., Bartholy, J., Beck, C., Erpicum, M., Esteban, P., Fettweis, X., Huth, R., James, P., Jourdain, S., Kreienkamp, F., Krennert, T., Lykoudis, S., Michalides, S. C., PiankoKluczynska, K., Post, P., Álvarez, D. R., Schiemann, R., Spekat, A., and Tymvios, F. S.: Cost733cat - A database of weather and circulation type classifications, Phys. Chem. Earth, 35, 360-373, 2010.

Pinson, P., Siebert, N., and Kariniotakis, G.: Forecasting of regional wind generation by a dynamic fuzzy-neural networks based upscaling approach, European Wind Energy Conference (EWEC), 2003.

Rockach, L.: A Survey of Clustering Algorithms, in: Data mining and knowledge discovery handbook, edited by: Maimon, O. and Rockach, L., 2 Edn., Springer, New York, 269-298, 2010.

Späth, S., von Bremen, L., Junk, C., and Heinemann, D.: Timeconsistent calibration of short-term regional wind power ensemble forecasts, Meteorol. Z., 24, 381-392, 2015. 
Trancoso, A. R. R.: Operational Modelling as a Tool in Wind Power Forecast and Meteorological Warnings, Doctoral Dissertation, Instituto Superior Técnico, Universidade Técnica de Lisboa, Lisboa, Portugal, 2012.

Trigo, R. and da Camara, C. C.: Circulation weather types and their influence on the precipitation regime in Portugal, Int. J. Climatol., 20, 1559-1581, 2000.
Vincent, C. L.: Mesoscale wind fluctuations over Danish waters, Doctoral Dissertation, Technical University of Denmark, Ris $\varnothing$ National Laboratory for Sustainable Energy, 2010. 